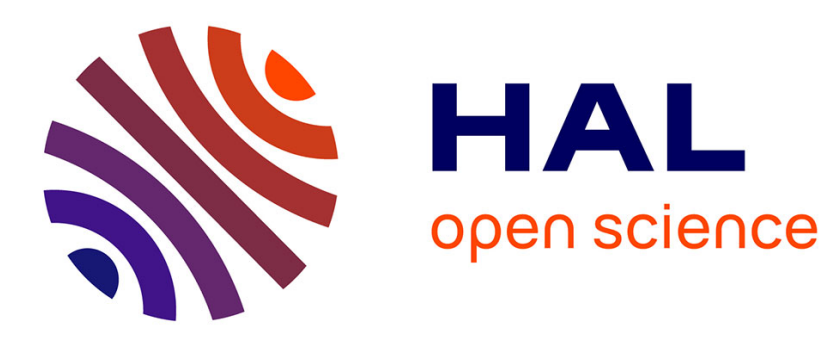

\title{
Segmental information drives adult bilingual phrase segmentation preference Authors
}

Irene de La Cruz-Pavía, Gorka Elordieta, Julián Villegas, Judit Gervain, Itziar Laka

\section{- To cite this version:}

Irene de La Cruz-Pavía, Gorka Elordieta, Julián Villegas, Judit Gervain, Itziar Laka. Segmental information drives adult bilingual phrase segmentation preference Authors. International Journal of Bilingual Education and Bilingualism, 2020, 10.1080/13670050.2020.1713045 . hal-02877149

\section{HAL Id: hal-02877149 \\ https://hal.science/hal-02877149}

Submitted on 22 Jun 2020

HAL is a multi-disciplinary open access archive for the deposit and dissemination of scientific research documents, whether they are published or not. The documents may come from teaching and research institutions in France or abroad, or from public or private research centers.
L'archive ouverte pluridisciplinaire $\mathbf{H A L}$, est destinée au dépôt et à la diffusion de documents scientifiques de niveau recherche, publiés ou non, émanant des établissements d'enseignement et de recherche français ou étrangers, des laboratoires publics ou privés. 
"This is an Accepted Manuscript of an article published by Taylor \& Francis Group in International Journal of Bilingual Education and Bilingualism on 22/01/2020, available online: https://www.tandfonline.com/doi/full/10.1080/13670050.2020.1713045.

\section{Segmental information drives adult bilingual phrase segmentation preference}

\section{Authors:}

Irene de la Cruz-Pavía1*, Gorka Elordieta1', Julián Villegas², Judit Gervain ${ }^{3,4}$, and Itziar Laka1

1Department of Linguistics and Basque Studies, University of the Basque Country UPV/EHU,

Vitoria-Gasteiz, Spain

${ }^{2}$ Computer Arts Laboratory, University of Aizu, Aizu-Wakamatsu, Japan

3 Integrative Neuroscience and Cognition Center (INCC — UMR 8002), Université Paris Descartes

(Sorbonne Paris Cité), Paris, France

${ }^{4}$ Integrative Neuroscience and Cognition Center (INCC — UMR 8002), CNRS, Paris, France

* Now at: Integrative Neuroscience and Cognition Center (INCC — UMR 8002), CNRS—Université Paris Descartes (Sorbonne Paris Cité), Paris, France

Corresponding author: Irene de la Cruz-Pavía idelacruzpavia@gmail.com 


\begin{abstract}
In two artificial language learning experiments with four groups of highly proficient Basque-Spanish bilinguals and two groups of Spanish monolinguals, we examine the cues that allow adult listeners to parse new input into phrases. In addition, we investigate which factors lead bilinguals to switch between the segmentation strategies characteristic of their two languages. We show that segmental information drives bilinguals' choice of a segmentation strategy when presented with an unfamiliar language. The language in which bilinguals are addressed during the study (i.e., the language of context) additionally modulates their segmentation preference, and this context language effect is found in L1Basque bilinguals but does not extend to L1Spanish bilinguals. The cause of this asymmetry is yet to be established. Finally, we show that adult monolinguals disregard statistical cues in favor of unfamiliar segmental information when in conflict. These results evidence that the available phrase segmentation cues are arranged hierarchically.
\end{abstract}

\title{
Keywords
}

Bilingualism, artificial language learning, phrase segmentation, segmental cues, statistical cues, context language effect 


\section{Introduction}

When bilingual speakers perceive or produce language, both of their languages are simultaneously activated (Colomé, 2001; Costa, Caramazza, \& Sebastián-Gallés, 2000; Green, 1998). Bilinguals thus need to constantly monitor their two systems - controlling or inhibiting the non-target language (Green, 1998) — and switch between them as a function of linguistic and non-linguistic factors (e.g., situational or pragmatic factors, Grosjean, 2001). The present study seeks to further our understanding of how bilinguals accomplish this feat. To do so, we examine the role and interplay of some of these potential cues in a pivotal aspect of speech processing, namely speech segmentation.

In order to process the structure of the input and retrieve its meaning, we must identify its components, locating the boundaries of morphemes, words, and phrases. The present study focuses on cues to the segmentation of units bigger than the word. We seek to determine the phrase segmentation cues ${ }^{1}$ available to adults — bilingual and monolingual - and explore which factors lead bilinguals to switch between the strategies characteristic of their two languages when parsing new input. In recent years, a handful of studies using the artificial language learning paradigm have investigated adult's abilities to extract phrases from unfamiliar languages. These studies have identified two sources of information that allow adults to parse this new input. Thus, specific types of distributional and prosodic information - i.e., word frequency and phrasal prosody $^{2}$ - provide reliable cues to phrase boundaries (Gervain, Nespor, Mazuka, Horie, \& Mehler, 2008; Nespor et al., 2008) and are available to adults (de la Cruz-Pavía, Werker, VatikiotisBateson, \& Gervain, 2019; Gervain et al., 2013). Two recent studies have investigated how adult

\footnotetext{
1 We use the terms segmentation and parse interchangeably throughout the paper. In the present study, we examine adults' abilities to extract phrases from new input, that is, to locate their boundaries. However, we do not wish to claim that the listeners process the semantic and syntactic information contained in these phrases.

2 With and without a concurrent visual facial correlate to auditory phrasal prosody, specifically head nodding (de la Cruz-Pavía et al., 2019).
} 
bilinguals exploit these cues to finding phrases in artificial languages (see de la Cruz-Pavía et al., 2015 for frequency-based cues, and de la Cruz-Pavía et al., 2019 for frequency-based as well as auditory and visual prosodic information). These studies have revealed enhanced performance in bilinguals as compared with monolinguals. That is, when segmenting new languages, bilinguals seem to rely on these cues to a greater extent than their monolingual peers (de la Cruz-Pavía et al., 2019). These findings tally with the bilingual advantage observed in the detection of other statistical regularities (Poepsel \& Weiss, 2016; Tsui, Erickson, Thiessen, \& Fennel, 2017; Wang \& Saffran, 2014), and in their use of visual facial information in other aspects of speech processing, such as language discrimination (Soto-Faraco et al., 2007; Weikum et al., 2013).

The present study focuses on one of these cues, namely, the relative frequency and order of functors and content words in natural languages. Functors - determiners, pronouns, adpositions, verbal inflection: the, she, on, jump-ed... - signal grammatical relations, form small and closed classes, and are perceptually minimal (Selkirk, 1996; Shi, Morgan, \& Allopenna, 1998). Conversely, content words - nouns, verbs, adjectives, etc.: chocolate, eat, slow - carry lexical meaning, form large and open classes, and are acoustically more salient (e.g., typically receive prosodic prominence; Selkirk, 1996; Shi et al., 1998). Statistically, individual functors have a high frequency of occurrence, whereas individual content words occur much less frequently in the input (Cutler, 1993; Gervain et al., 2013). Importantly, functors tend to occur at the edges of syntactic phrases (Gervain et al., 2013), and the linear order of functors and content words varies crosslinguistically in correlation with the basic order of Verbs and Objects. In V(erb)-O(bject) languages (e.g., Spanish, English), functors typically occur phrase-initially (e.g., English: of the woman; Spanish: de la mujer), while in $\mathrm{O}($ bject)-V(erb) languages (e.g., Basque, Japanese), functors tend to appear phrase-finally instead (e.g., Basque: emakume-a-ren woman-the-of; Japanese: Onna-no woman-of). 
The relative frequency and distribution of functors and content words is therefore a cue to phrase boundaries and basic word order. Humans are sensitive to this cue from early stages of development. When presented with artificial languages comprised of strictly alternating frequent and infrequent elements (mirroring this frequency-based property of functors and content words, respectively) such that the artificial language has an ambiguous structure, both adults and prelexical infants parse this new input into phrase-like units that follow the order of functors and content words in their native languages (adults: de la Cruz-Pavía et al., 2019; Gervain et al., 2013; infants: Gervain et al., 2008; Marino, Bernard, \& Gervain, accepted).

A recent study by de la Cruz-Pavía et al. (2015) investigated whether adult bilinguals, speakers of a VO and an OV language, could exploit the frequency-based strategies characteristic of their two languages. They presented highly proficient bilinguals, speakers of Basque (OV, functor-final language) and Spanish (VO, functor-initial language), with an artificial language (AL) composed of elements that occurred either frequently (fi, nu, ge) or infrequently (LU, FE, MU, TA, etc.) and were presented in strict alternation (see Figure 1 for details of an AL with a similar structure). The beginning and end of the stream were faded in and out, rendering the language structurally ambiguous, with two possible parses: (i) a frequent-initial parse, characteristic of VO languages (e.g., Spanish; ...fiLUnuFEgeMUfiPEnuTAgeLI...), or (ii) a frequent-final parse, characteristic of OV languages (e.g., Basque; ...LUnuFEgeMUfiPEnuTAgeLlfi...). After listening to the artificial language for 17 minutes, participants were presented with pairs containing a frequentinitial and a frequent-final sequence - each six-syllable long - and had to choose, for each pair, the sequence they thought belonged to the artificial language. All bilinguals were presented with the same language. They were sorted into four groups on the basis of (i) their linguistic background, and (ii) the language in which they received the instructions and were addressed during the study, i.e., the language of context. Thus, two of the groups had acquired Basque as 
their first language (L1) and Spanish as their second language (L2), while the remaining two groups had acquired Spanish as their L1, and Basque as their L2. In turn, one of the L1Basque and one of the L1Spanish groups were addressed exclusively in Basque during the study, while the remaining two groups of bilinguals were addressed exclusively in Spanish.

The four groups of bilinguals had a preference towards parsing the artificial language into a frequent-final order, the order characteristic of Basque, their OV language (their means are reported in Table 1). However, the language of context significantly impacted participants' segmentation preference: bilinguals had a stronger frequent-final (i.e., functor-final) segmentation of the language when addressed in Basque (OV), than when addressed in Spanish (VO). This Context Language Effect was restricted to the two groups of L1Basque bilinguals, and not found in the groups of L1Spanish bilinguals. These results suggest that (certain) bilinguals are able to deploy the segmentation strategies of their two languages. Moreover, this research uncovered a new factor - context language -that influences bilinguals' segmentation preferences. Interestingly, changing the language of context did not result in a full reversal of their segmentation preference, but rather modulated the degree of their preference for a frequent-final order.

The artificial language had been synthesized using a German voice from MBROLA (Dutoit, 1997), as this language was unknown to the bilinguals tested. De la Cruz-Pavía et al. (2015) argued that the segmental (e.g., acoustic-phonetic) information contained in the German voice could have overridden the impact of the language of context, biasing participants towards a frequent-final segmentation preference. Specifically, de la Cruz-Pavía et al. (2015) proposed that acoustic-phonetic differences in the realization of stops in German vs. Spanish and Basque might be at the origin of this bias, though this hypothesis is yet to be confirmed (this claim is further developed in the general discussion section). 
In the present study, we put this hypothesis to test, investigating for the first time the relative role and interplay of these two cues - language of context and segmental information in adult bilinguals' ability to parse new input into phrases. Note that with the term segmental, we comprise all potential sources of information at and below the segment level (i.e. phonemic and subphonemic cues, respectively). Segmental information has been shown to play an important role in speech segmentation at the word level. Adult listeners make use of minimally three segmental cues - phonemic and subphonemic - to extract words from the input: (a) phonotactic constraints, that is, the legal phoneme sequences and their relative frequency of occurrence in a given language (phonemic cue; Finn \& Hudson Kam, 2008), (b) allophonic variation, that is, contextdependent variants of a given phoneme (subphonemic cue; Smith \& Hawkins, 2000), and (c) coarticulation, that is, the overlap of adjacent articulations while producing speech sounds (subphonemic cue; Mattys, White, \& Melhorn, 2005). Moreover, adult listeners make preferential use of these segmental cues to words, and relegate other sources of information such as statistical and prosodic cues to a less salient position (Mattys et al., 2005; Finn \& Hudson Kam, 2008; Mersad \& Nazzi, 2011).

If the general frequent-final segmentation preference obtained in de la Cruz-Pavía et al. (2015) indeed resulted from the segmental properties of the German voice used to synthesize the artificial language, this would suggest that, in the panoply of phrase segmentation cues available to bilinguals, segmental cues outweigh language of context. We hypothesize that removing potentially biasing segmental information might lead adult bilinguals to fully rely on the language of context to segment new input. In other words, if bilinguals are presented with an AL that contains segmental information found in both their languages, which thus renders the $\mathrm{AL}$ ambiguous between the languages at the segmental level, bilinguals might reverse their segmentation preferences of this new AL as a function of the language of context. Spanish and Basque spoken in Spain (languages 
tested in de la Cruz-Pavía et al., 2015) are ideal candidates to test this hypothesis, as their sound systems greatly overlap. Both languages have the same vocalic inventory, and the Spanish consonantal set is a subset of the Basque inventory, with very few exceptions (e.g., the fricative $/ \theta /$; Hualde, 2003, 2005). Furthermore, these two languages share a substantial part of their phonotactic constraints (Hualde, 2003, 2005).

In a first experiment (Experiment 1), we examine the segmentation preferences of four groups of Basque-Spanish bilinguals of an AL that has an ambiguous structure that allows both a frequent-initial and a frequent-final parse — identical to the one in de la Cruz-Pavía et al. (2015) — in addition to segmental information ambiguous between Basque and Spanish. As in de la CruzPavía et al. (2015), we sorted bilinguals into four groups depending on their L1 (Basque or Spanish), and the language in which they were addressed during the study (i.e., the language of context: Basque or Spanish). If, in the face of ambiguous segmental and statistical information, the language of context now determines bilinguals' segmentation of the $\mathrm{AL}$, a frequent-initial segmentation preference of the AL is expected to obtain in the participants addressed in Spanish, while the reverse frequent-final segmentation preference is predicted in the bilinguals addressed in Basque. In addition, testing L1Basque and L1Spanish bilinguals will allow us to explore potential differences between these two populations. Specifically, we will determine whether the predicted context language effect is smaller or absent in the two groups of L1Spanish bilinguals, as found in de la Cruz-Pavía et al. (2015).

In a second experiment (Experiment 2), we further examine the role of segmental information, focusing now on the segmentation abilities of adult monolinguals. Thus, we investigate the interplay of this source of information and frequency-based cues, in order to determine whether segmental information outweighs statistical information, as found in speech segmentation at the 
word-level (Finn \& Hudson Kam, 2008; Mersad \& Nazzi, 2011). To do so, we examine the segmentation preferences of two groups of monolingual speakers of Spanish (VO language), each

presented either with Experiment 1's segmentally-ambiguous AL, or with the original AL in de la Cruz-Pavía et al. (2015), synthesized using a German voice.

\section{Experiment 1}

\section{Methods}

\section{Participants}

Ninety-two students (71 females, mean age 20 , age range 18 to 31 ) from the University of the Basque Country UPV/EHU (Vitoria-Gasteiz, Spain) took part in this experiment. Following de la Cruz-Pavía et al. (2015), we sorted participants into four groups, depending on their native language and the language in which they received the instructions (i.e., context language). Twentyseven L1Basque-L2Spanish bilinguals (19 females, mean age 20, age range 18 to 31 ) were addressed and received the instructions in Basque, while another 23 L1Basque-L2Spanish bilinguals (17 females, mean age 18, age range 18 to 18 ) were addressed in Spanish. Twenty-one L1Spanish-L2Basque bilinguals (15 females, mean age 21 , age range 18 to 26 ) participated in the experiment conducted in Basque, and another 21 L1Spanish-L2Basque bilinguals (20 females, mean age 21, age range 18 to 27) in the experiment conducted in Spanish.

In order to determine their linguistic background, participants filled the same questionnaire described in de la Cruz-Pavía et al. (2015, reproduced in their Appendix I). This questionnaire was developed by members of the research group The Bilingual Mind (UPV/EHU), and measures use of the bilinguals' two languages in different contexts and at three different points of development. To ensure comparability, we chose bilinguals that had similar backgrounds to the participants in de la Cruz-Pavía et al. (2015). Thus, all L1Basque bilinguals had been raised in Basque-speaking 
homes minimally until adolescence. In turn, L1Spanish bilinguals had been raised in Spanishspeaking homes until adolescence. Average age of acquisition (AoA) of the $L 2$ in the four groups was between 3 and 5 years. All bilinguals had had formal education in both their languages and reported being highly proficient in them. Participants received a small compensation for their participation.

\section{Stimuli}

We created an artificial language (AL) with an ambiguous structure similar to the ALs in Gervain et al. (2013) and de la Cruz-Pavía et al. $(2015,2019)$. This AL consisted of three frequent categories: $a, b$ and $c$, and three infrequent categories: $X, Y$ and $Z$ (see Figure 1). Each frequent category contained a single $\mathrm{C}$ (onsonant)- $\mathrm{V}$ (owel) token, while infrequent categories contained $9 \mathrm{CV}$ tokens each. Therefore, the AL comprised 3 frequent tokens and 27 infrequent tokens. We combined the six categories into an $a X b Y c Z$ basic unit. As a result, frequent and infrequent categories were in strict alternation, and tokens from frequent categories occurred hence 9 times more frequently than tokens from infrequent categories.

\section{[INSERT FIGURE 1 HERE]}

We concatenated the six-syllable basic unit 377 times (aXbYcZaXbYcZaXbYc...), creating a 9 minute and 3 second long familiarization stream. As frequent categories contained a single token each, their order in the stream remained constant (fiXnuYpeZfiXnuYpeZfiXnuYpeZ...). Meanwhile, the 9 tokens of each infrequent category occurred randomly (fiNEnuLIpeKIfiKAnuLOpeLEfiKAnuPUpeFO...). We faded in and out the stream to render it 
ambiguous. The resulting stream allowed two possible segmentations: (a) a frequent-initial segmentation: [aXbYcZ]aXb..., or (b) a frequent-final segmentation: $a\left[X b Y_{c} Z a\right] X b \ldots$.

As in de la Cruz-Pavía et al. (2015), test stimuli consisted of 36 six-syllable long items. Half of the items started with a frequent category (e.g., bYcZaX: nuLIpeKUfiTO), that is, they had a frequent-initial order. The remaining half started with an infrequent category (e.g., YcZaXb:SApeKEfiMUnu) and had hence a frequent-final order. All categories (frequent and infrequent) occurred with similar frequency in all possible positions.

In order to provide the AL with ambiguous segmental information, we created a new lexicon for the AL that differed from the one in de la Cruz-Pavía et al. (2015). The phonetic inventories of Basque and Spanish overlap largely, which allowed us to build a lexicon comprised only of phonemes shared in the two languages (see Figure 1). We took into consideration the phonotactics and allophonic distribution characteristic of Basque and Spanish (see Appendix 1 for further detail), as adult listeners use these two cues to segment speech (Finn \& Hudson Kam, 2008; Mersad \& Nazzi, 2011; Smith \& Hawkins, 2000). Additionally, test items and familiarization were synthesized with the es1 (Spanish male) voice of the MBROLA database (Dutoit, 1997), instead of the German voice (de6, German male) used in de la Cruz-Pavía et al. (2015). Note that there are currently no MBROLA voices available in Basque. As in de la Cruz-Pavía et al. (2015), all segments in both the familiarization stream and test items were $120 \mathrm{~ms}$ long and had a monotonous fo of $100 \mathrm{~Hz}$, and a flat intensity level. The familiarization stream and test items are available in the Supplemental Materials.

\section{Procedure}

Participants were tested in the psycholinguistics laboratory at the University of the Basque Country (Vitoria-Gasteiz, Spain). At the beginning of the session, participants filled the language 
background questionnaire. All participants were tested individually in a quiet room, and provided with a set of high quality Logitech headphones. The experimental input was displayed in a computer screen using DMDX software (Forster \& Forster, 2003). Participants were told that they would first listen to an unknown language for about 9 minutes and that they needed to pay close attention, as they would then listen to questions regarding this language. Before the study, a short training was carried out, in order to familiarize participants with the procedure of the experiment. During training, participants heard six pairs of monosyllables (e.g., "me so") and had to identify a target syllable (i.e., "so") in every pair. They were asked to press one of two predefined keys in a keyboard, depending on whether the target syllable had been heard first, or second.

After training, participants listened to the 9 minute long familiarization followed by the test phase. During test, participants listened to a total of 36 trials. Each trial consisted of two six-syllable long sequences separated by a $500 \mathrm{~ms}$ silence. One of the sequences had a frequent-initial order, the other a frequent-final order, and order of presentation across trials was counterbalanced. Each test sequence appeared twice during the test phase, as first (e.g., fiTInuTEpeKUFUpeTAfiNEnu) and second (e.g., TAfiMUnuLIpe-fiTInuTEpeKU) member of a pair, but never in consecutive test trials. Participants had to choose the sequence in each pair that they thought belonged to the language heard in familiarization by pressing one of the two predefined keys in the keyboard. The session lasted around 30 minutes.

\section{Results and Discussion}

The computer recorded the number of frequent-final responses per participant out of the 36 test trials. To analyze the participants' responses we conducted binomial tests of proportions, given that participants' responses were binomial in nature, i.e., a two-alternative forced choice between frequent-initial and frequent-final items. This analysis allows us to directly compare with previous 
literature (de la Cruz-Pavía et al., 2015, 2019).

Within-group binomial tests of proportions revealed that all four groups had a frequentinitial parsing preference that differed significantly from chance (all $p<.001$; analyses were conducted in R, version 3.5.3., R Core Team 2019), as shown in Table 1 and Figure 2. Subject-bysubject counts are provided in Appendix 2. We submitted the responses of the four groups of bilinguals to a binomial test of proportions, which revealed a significant difference between them $\left(X^{2}(3, N=92)=10, p=.007\right)$. Pair-wise comparison of proportions with the Holm-Bonferroni method for $p$-value adjustment showed a single significant comparison: a significant difference in the responses of the two groups of L1Basque bilinguals $(p=.004$, Cohen's $h=.165$; all other comparisons $p \geq$.129). Thus, the group of L1Basque bilinguals addressed in Basque had a greater number of frequent-final responses $(13.67 / 36,37.96 \%)$ than the group of L1Basque bilinguals addressed in Spanish (10.87/36, 30.19\%).

These results reveal a general frequent-initial segmentation preference of the artificial language, which is modulated by the language of context in L1Basque bilinguals. Our goal in the present study was to provide Basque-Spanish bilinguals with ambiguous segmental cues, in order to examine the effect of the language of context in their parse of the AL. We predicted that, in the absence of other cues, the language of context would determine their segmentation preference. The results of Experiment 1 did not fulfill this prediction. Further, the general frequent-initial preference observed contrasts strongly with the general frequent-final segmentation reported in de la Cruz-Pavía et al. (2015) in four similar groups of bilinguals. The ALs in de la Cruz-Pavía et al. (2015) and the present study have identical structures but differ in their segmental information, that is, their lexicons as well as the voice in which they were synthesized (German vs. Spanish). The opposite segmentation preferences observed in de la Cruz-Pavía et al. (2015) and the present 
study suggest that, despite our attempt to render all segmental information ambiguous, phonemic and/or subphonemic segmental cues might have determined bilinguals' segmentation preference.

The present study also differed from the one in de la Cruz-Pavía et al. (2015) in another aspect, namely, the duration of familiarization. In the current study, we reduced familiarization length from 17 minutes and 30 seconds (in de la Cruz-Pavía et al., 2015), to 9 minutes and 3 seconds. In order to ensure that this change did not impact the participants' segmentation preferences, we conducted a control study with 12 L1Basque-L2Spanish bilinguals (11 females, mean age 19 , age range 18 to 22 ) that had the same language background as all other L1Basque participants. We familiarized this control group with an identical version of the present AL except for familiarization duration, which was now 17 minutes 4 seconds. This control group of bilinguals was addressed in Basque during the experiment. A within-group binomial revealed a frequent-initial segmentation preference that differed significantly from chance (number of frequent-final responses: $13.75 / 36, p<.001$; see Table 1 and Figure 2). We then compared the segmentation preferences of this control group with the corresponding group of L1Basque-L2Spanish bilinguals (i.e., context Basque) reported above. A binomial test of proportions showed that there was no significant difference between the two groups $\left(X^{2}(1, N=39)=.0005, p=1.0\right)$. These results show that reducing the familiarization duration did not impact participants' segmentation preference of the AL. Therefore, the frequent-initial preference obtained in all groups in all likelihood results from the change in the AL's segmental information.

[INSERT TABLE 1 HERE]

[INSERT FIGURE 2 HERE] 
As mentioned above, the language of context significantly modulated the segmentation preferences of L1Basque-L2Spanish bilinguals. This pattern of results replicates the context language effect obtained in de la Cruz-Pavía et al. (2015), and provides mounting evidence for the need to control this factor in studies involving bilingual populations. Interestingly, we also replicated the asymmetry in the responses of L1Basque and L1Spanish bilinguals originally reported in de la Cruz-Pavía et al. (2015), as language of context did not modulate L1Spanish bilinguals' segmentation of the AL. Though the origin of this asymmetry is yet to be determined, we discuss in what follows the potential role of two factors, i.e., proficiency and frequency of language use.

The asymmetry could plausibly stem from differences in the two populations' proficiency in their corresponding L2, if L1Spanish bilinguals had lower proficiency in Basque than L1Basque bilinguals in Spanish. In the present study, we did not measure proficiency by means of standardized assessment tests. However, we collected self-report ratings of the bilinguals' L2 proficiency speaking and understanding their L2 (8-point Likert scale, means are presented in Table 2 below). We explored these ratings to determine whether differences in reported L2 proficiency levels accounted — at least partially — for this asymmetry.

\section{[INSERT TABLE 2 HERE]}

We ran a cumulative link model fitted with Laplace approximation (ordinal package, Christensen, 2019) to examine the bilinguals' ratings of $L 2$ proficiency across groups. We built the simplest possible model and step-by-step added the factors Language of Context (Basque or Spanish) and L1 (Basque and Spanish), running ANOVAs to compare pairs of models. Random effects were not included, as each subject provided a single rating. Separate models were run with the ratings provided for the two skills, namely speaking and understanding. The models that best fit 
the ratings in both skills contained the fixed factor of $L 1$, but not the factor Language of Context. Indeed, a significant fixed effect of L1 was found in both models (speaking: $\beta=-0.391, \mathrm{SE}=0.196$, $z=-1.99, p=.046$, understanding: $\beta=-0.52, \mathrm{SE}=0.21, z=-2.48, p=.013)$. This effect reflected the fact that L1Spanish bilinguals reported greater L2 proficiency than L1Basque bilinguals, in speaking as well as understanding. As expected, the factor Language of Context did not significantly account for part of the variability in the dataset. This suggests that both L1Basque groups reported similar levels of proficiency in their respective $L 2$, and so did both groups of L1Spanish.

To further investigate a potential impact of proficiency in the bilinguals' parsing of the AL, we examined the relationship between reported L2 proficiency (in speaking and understanding) and their segmentation preference of the $\mathrm{AL}$ (i.e., the number of frequent-final responses in test) in each of the four groups of bilinguals. To quantify potential correlations, we computed Spearman rank order correlation coefficients. The results of this analysis revealed no significant correlation in any of the four groups (speaking: all $p \geq .359, r_{s}=-.184$ to .191 ; understanding: all $p \geq .150, r_{s}=-$ 196 to .326$)$.

In sum, L1Spanish bilinguals reported greater proficiency in L2 than L1Basque bilinguals, both groups of L1Spanish bilinguals reported similar proficiency ratings, and so did both groups of L1Basque bilinguals. Finally, no correlation obtained between reported proficiency and segmentation preference of the AL. Taken together, these results suggest that the absence of a context language effect found in L1Spanish bilinguals did not stem from lower reported proficiency in their L2.

Differences in frequency of language use could potentially be another cause for the observed asymmetry between L1Spanish and L1Basque bilinguals, if L1Basque bilinguals have a more balanced use of their two languages. We only recruited participants who had been raised in 
either monolingual Basque- or monolingual Spanish-speaking homes (L1Basque and L1Spanish participants, respectively), and ensured that all participants had received formal bilingual education and were highly proficient in Basque and Spanish. In order to obtain a measure of language use, we asked participants to rate the relative frequency in which they used their two languages in everyday life, in situations other than at home and at educational settings. They again provided separate ratings (7-point Likert scale, see Table 2) for infancy, adolescence and adulthood. Participants reported using their respective L1 predominantly in their interactions (average of the two groups at the three developmental points: 2.21 in L1Basque bilinguals, 5.12 in L1Spanish, where 1 means use of Basque-only, and 7 use of Spanish-only). We investigated a potential association between number of frequent-final responses and frequency of language use in the three points in development, for each of the four groups of bilinguals. The results of these analyses revealed no significant correlation, with one exception, namely a positive correlation between reported frequency of use and number of frequent-final responses in the group of L1Spanish bilinguals tested in a Basque context, and only in adulthood $\left(r_{s}=.451, p=.040\right.$; all other $p \geq .129$ and $r_{s}=-341$ to 221 ). Note that higher scores in reported language frequency depict greater use of Spanish and lesser use of Basque. Taken together, these results do not suggest a strong relationship between language use and bilingual speech segmentation. Nonetheless, they open the ground for more in depth investigations on the potential role of frequency of language use - in particular at the time of testing - in the absence of a context language effect found in L1Spanish bilinguals.

In sum, the results of Experiment 1 show that the language of context modulated but did not determine bilinguals' segmentation of the AL. The frequent-initial preference obtained in all groups might instead result from changes in segmental information between the present $A L$ and the AL in de la Cruz-Pavía et al. (2015). Participants were bilingual speakers of a VO (Spanish) 
and an OV (Basque) language, and are hence exposed to both frequent-initial and frequent-final constituents in their input. As a result, frequency-based information is an ambiguous cue for these VO-OV bilinguals. This information is not ambiguous for monolingual speakers. The relative weight of segmental information as a cue to parsing the input might hence differ between VO-OV bilinguals and monolinguals. In Experiment 2 we further explore the relative role and interplay of segmental and frequency-based information, by examining the segmentation preferences of two groups of Spanish monolinguals exposed to ALs with different segmental information.

\section{Experiment 2}

Does segmental information determine monolingual's segmentation preference of an AL? To answer this question, we present two groups of Spanish monolinguals with (a) the "Spanish" AL tested in Experiment 1 and (b) the original "German" AL in de la Cruz-Pavía et al. (2015). While the former was parsed into a frequent-initial word order by four groups of Basque-Spanish bilinguals, four similar groups segmented the latter into the opposite frequent-final parse. Given that both languages share the exact same frequency-based information, the bilinguals' segmentation might have been driven by the differing segmental information in the two ALs.

A frequent-initial segmentation preference is predicted in the group of Spanish monolinguals exposed to Experiment 1's Spanish AL. Frequency-based information signals a frequent-initial word order to these speakers of a VO language. Furthermore, the AL contains segmental information characteristic of the native language and is synthesized using a Spanish voice. Meanwhile, no clear preference is predicted in the group of Spanish monolinguals exposed to the German AL in de la Cruz-Pavía et al. (2015). A frequent-initial segmentation would evidence that Spanish monolinguals disregard the unfamiliar segmental information, relying instead in the

available frequency-based cues. A frequent-final segmentation would instead suggest that 
segmental information, even if unfamiliar, determines monolinguals' parse of the AL, overriding the familiar frequency-based cue. Finally, an absence of preference would suggest that monolingual speakers weigh these two cues equally.

\section{Methods}

\section{Participants}

Fifty-six Spanish monolinguals (40 females, mean age 25 , age range 18 to 41 ) took part in Experiment 2. Of these, a group of 27 Spanish monolinguals (21 females, mean age 25 , age range 18 to 41) were presented with the Spanish AL from Experiment 1. A further group of 29 Spanish monolinguals (19 females, mean age of 26 , range 18 to 41 ) were exposed to the original, German AL from de la Cruz-Pavía et al. (2015), presented in Figure 3. All participants reported minimal or no knowledge of Basque.

\section{Stimuli}

As described in Experiment 1, the two artificial languages shared the same basic structure and differed only in two aspects: their lexicon, and the voice with which they were synthesized. Thus, the Spanish and German ALs had different CV tokens (listed in Figures 1 and 3 respectively). The Spanish AL was synthesized using the es1 Spanish voice from MBROLA, while the German AL was synthesized using the de6 German voice, from the same database. As the familiarization streams were the ones originally used in de la Cruz-Pavía et al. (2015) and Experiment 1, the German stream was 17 min long, whereas the Spanish stream was 9 min long. In all other respects the two ALs were identical (see Experiment 1 for a detailed description). The familiarization stream and test items are available in the Supplemental Materials. 


\section{Procedure}

All participants were tested in the psycholinguistics laboratory at the University of the Basque Country (Vitoria-Gasteiz, Spain). Procedure was identical to the one described in Experiment 1.

[INSERT FIGURE 3 HERE]

\section{Results and Discussion}

Figure 4 and Table 1 show the number of frequent-final responses per group out of the 36 trials. Within-group binomial tests of proportions revealed that both groups had a parsing preference that differed significantly from change (both $p<.001$ ). Importantly, the two groups displayed opposite preferences. The group exposed to the German AL segmented the language into a frequent-final pattern (frequent-final responses: 25/36, 69.44\%). Meanwhile, the group exposed to the Spanish AL segmented the language into a frequent-initial pattern (frequent-final responses: 14.7/36, 40.84\%). A binomial test of proportions revealed a significant difference in the responses of the two groups $\left(X^{2}(1, N=56)=200, p<.001\right.$, Cohen's $\left.h=.584\right)$. In sum, we found a total reversal of their segmentation preferences as a function of the segmental information in the artificial language.

\section{[INSERT FIGURE 4 HERE]}

\section{General Discussion}

The interplay of language of context and segmental information in bilingual speech segmentation 
The present research investigated the factors leading adult bilinguals to deploy the strategies of their two languages and switch between them when parsing new input. To do so, we focused on a pivotal aspect of speech processing, namely speech segmentation. We examined the role of a pragmatic factor - the language of context - and its interplay with segmental information, in bilinguals' parsing of an unfamiliar artificial language. In particular, we sought to establish whether, when presented with ambiguous segmental information, the language of context (i.e., the language in which participants were addressed during the experiment), would determine adult bilinguals' choice of a strategy to parse new input.

In Experiment 1, we examined the segmentation preferences of an artificial language, of bilingual speakers of Basque (OV) and Spanish (VO), sorted into four groups as a function of their L1 (Basque or Spanish), and the language of context (Basque or Spanish). The AL consisted of strictly alternating frequent and infrequent elements, mimicking this frequency-based property of functors and content words, respectively, in natural languages. Crucially, the AL had an ambiguous structure that allowed two possible segmentations depending on the ordering of its elements. These two possible segmentations mirrored the opposite word orders characteristic of Basque (functor/frequent-final language: emakume-a "woman the") and Spanish (functor/frequent-initial language: la mujer "the woman"). Moreover, the AL consisted of phonemes present both in the Spanish and Basque sound systems, and the resulting lexicon respected the phonotactics of the two languages. Therefore, the AL provided ambiguous segmental and frequency-based information to the OV-VO bilinguals. We hypothesized that bilinguals might rely on the sole available cue, i.e., language of the context, to parse this new language. We hence predicted opposite segmentation preferences of the same AL in the bilinguals addressed in Basque vs. Spanish during the study.

This prediction was not borne out by the results of Experiment 1, as all four groups of bilinguals segmented the AL into a frequent-initial word order, order characteristic in their VO 
language, Spanish. However, a context language effect obtained in the two groups of L1Basque bilinguals. When addressed in Basque, L1Basque bilinguals chose a significantly greater frequentfinal segmentation of the language (13.67 frequent-final responses out of 36 trials, $37.96 \%)$ than when addressed in Spanish (10.87/36 frequent-final responses, 30.19\%). No such effect obtained in the segmentation preferences of the two groups of L1Spanish bilinguals (context Basque: 12.81/36, 35.58\%, context Spanish: $12.52 / 36,34.79 \%)$. This pattern of results replicates exactly the results obtained in de la Cruz-Pavía et al. (2015) with four similar groups of bilinguals, evidencing that the language of context modulated but did not determine their parsing of the AL.

While the ALs in de la Cruz-Pavía et al. (2015) and in the present research were structurally identical and contained the exact same frequency-based information, they differed in their segmental information. Thus, the AL in de la Cruz-Pavía et al. (2015) was synthesized using a German voice from the MBROLA database (Dutoit, 1997). Meanwhile, in the present experiment, we created a new lexicon that exclusively comprised segments shared in the Spanish and Basque phonologies, and synthesized the AL using a Spanish voice. In de la Cruz-Pavía et al. (2015), all four groups of bilinguals - sorted by L1 and language of context - segmented the German AL into a frequent-final word order, whereas four similar groups of bilinguals segmented the Spanish AL in Experiment 1 into the reverse frequent-initial word order. Taken together, the results in de la Cruz-Pavia et al. (2015) and the present research show that the bilinguals' segmentation was mainly determined by the segmental information present in the input. Furthermore, these results suggest that segmental cues outweigh the cue provided by the language of context, though adult bilinguals reveal sensitivity to both sources of information. This pattern supports thus a hierarchical arrangement of phrase segmentation cues, as proposed by de la Cruz-Pavía et al. $(2015,2019)$, and similar to what is found in word segmentation cues (Mattys et al., 2005). 
What are the specific segmental cues responsible for this reversal in the bilinguals' segmentation preference? As mentioned, all segments in the present AL were part of both the Basque and Spanish sound inventories and the lexicon followed the phonotactics characteristic of these two languages. The resulting stream could therefore be interpreted as belonging to either of the two languages. However, as the Spanish consonantal set is - with very few exceptions - a subset of the Basque inventory, participants might have interpreted the absence of Basque-specific consonants (e.g. / , tș, ts /), which occur frequently in the input, as a cue towards deploying the strategies characteristic of Spanish. Further, we synthesized the stream using a Spanish voice from the MBROLA database (es1). Despite the absence of prosodic information (the stream had constant pitch and segment duration) acoustic-phonetic details in the realization of these sounds might also have biased participants towards relying on the strategies characteristic of Spanish when segmenting the AL. Unfortunately, we cannot currently test this hypothesis, as the MBROLA database contains no Basque voice. Note however that the voice used to synthesize the stream might have contributed but cannot fully account for the general frequent-initial segmentation preference obtained. Thus, Gervain et al. (2013) presented twelve L1Basque bilinguals - due to the impossibility to find adult Basque monolinguals — with a structurally identical artificial language that had been synthesized using the same Spanish es1 voice from MBROLA. The AL in Gervain et al. (2013) differed from the current one solely in their inventory of segments and lexicon, that is, in their set of CV tokens. Interestingly, the L1Basque bilinguals strongly preferred a frequent-final segmentation of the AL $(25.75 / 36,71.53 \%)$. These conflicting results suggest that bilinguals are highly sensitive to the particular set of segments and/or CV tokens that comprise the ALs, and opens the ground for future studies investigating the specific segmental cues - phonemic and subphonemic - driving bilinguals' segmentation, as well as their relative role and interplay. Adult 
listeners can make use of phonotactic constraints (phonemic cue, Finn \& Hudson Kam, 2008), allophonic variation (subphonemic cue, Smith \& Hawkins, 2000), and coarticulation (subphonemic cue, Mattys et al., 2005) to parse new input. It is hence likely that these sources of information allow bilinguals to choose between the strategies of their two languages when facing new input.

Experiment 1 revealed an asymmetric effect of language of context, replicating the results obtained in de la Cruz-Pavía et al. (2015). Thus, the language in which participants were addressed and received the instructions of the study significantly modulated L1Basque bilinguals' segmentation of the AL, but did not impact L1Spanish bilinguals' segmentation. We hypothesized that this asymmetry might result from differences in proficiency, if L1Spanish bilinguals had lower proficiency in their L2 - Basque - and could hence not switch between the strategies of their two languages. Participants filled a language questionnaire before the study, in which they rated their proficiency and language use of Basque and Spanish. Analysis of the participants' self-reported proficiency revealed that, contrary to our prediction, L1Spanish bilinguals reported significantly greater proficiency in their L2 (both in speaking and understanding), than L1Basque bilinguals. This result, added to the absence of a correlation between the bilinguals' segmentation preference and level of self-reported L2 proficiency, suggests that the origin of the asymmetry found between L1Basque and L1Spanish bilinguals might lie elsewhere. Note however that, though self-reported proficiency has been shown to be predictive of language ability (Luk \& Bialystok, 2013; Marian, Blumenfeld, \& Kaushanskaya, 2007), self-assessments of L2 proficiency are not direct, objective measurements of proficiency. Further, differences in response styles have been reported across countries as well as across cultures within countries, likely due to identitary factors. Thus, certain cultures/populations tend to choose extreme scores for the same stimuli (e.g., a 7 in a 7-point Likert scale), while others are more prone to choosing more middle responses (e.g., a 5 in the same scale, Harzing, 2006). 
Self-rated proficiency, proficiency determined via standardized tests, and language use, have been shown to be highly correlated, and be crucial dimensions of the "bilingual experience" (Luk \& Bialystok, 2013). We hence put forward language use as another possible factor behind the observed asymmetry. We hypothesized that the context language effect found in the two groups of L1Basque bilinguals might potentially result from a more balanced use of their two languages, as compared with L1Spanish bilinguals. We asked participants to rate the relative frequency in which they used their two languages in contexts other than at home and at educational settings. Both L1Basque and L1Spanish participants reported predominant use of their respective L1, i.e., in around $75 \%$ or more of their interactions. The present bilinguals had thus a pattern of language use similar to the ones tested in de la Cruz-Pavía et al. (2015). We again explored a potential relation between the bilinguals' language use and their segmentation preferences. A correlation emerged only in the group of L1Spanish bilinguals tested in Basque. Interestingly, this correlation was limited to their language use in adulthood. This result is too weak to draw any conclusion, but reveals the need to examine the role of language use in the segmentation abilities of adult bilinguals more systematically.

We put forward a third and closely related dimension of bilingualism that could have contributed to the asymmetry obtained in the segmentation preferences of L1Basque and L1Spanish bilinguals, namely the frequency of code-and/or language-switching. We hypothesize that the frequency with which bilinguals spontaneously switch from one of the bilingual's languages to the other within an interaction, or alternate between their languages in daily life, could lead them to more flexibly switch between the strategies from their two languages. Bilinguals that switch languages frequently have been shown to outperform less frequent language switchers both in linguistic and non-linguistic tasks (Prior \& Gollan, 2011; Verreyt, Woumans, Vandelanotte, Szmalec, \& Duyck, 2015; Yim \& Bialystok, 2012; but see Paap et al., 2016). Unfortunately, the 
linguistic background questionnaire filled by the present bilinguals did not ask about their language switching habits.

Finally, the asymmetric occurrence of the context language effect could be caused by language distance - operationalized as the degree of similarity of two languages' grammars - if the strategy characteristic of OV languages (i.e., Basque) can only be acquired as part of the L1's repertoire of processing strategies. A growing body of literature is documenting language distance effects in L2 processing. This research suggests that grammatical features absent in the bilinguals' L1 are harder to learn despite early Age of Acquisition and high proficiency (Díaz et al., 2016; Erdocia, Zawiszewski, \& Laka, 2014; Zawiszewski, Gutiérrez, Fernández, \& Laka, 2011). However, which features of language are affected by this factor remains to be determined. It is therefore possible that L1Spanish bilinguals have not acquired the strategy of their L2 and can only deploy the L1's segmentation strategy. These bilinguals would thus deploy the frequent-initial segmentation strategy characteristic of their L1, with no modulation of the language of context. In turn, the preference for a frequent-final parse observed in the same population in de la Cruz-Pavía et al. (2015), would result from the bias induced by specific segmental information of the German AL, which will be further discussed in the next section.

Future studies will determine whether the observed asymmetry results from differences in language proficiency and use habits - including patterns of language switching - or is instead caused by language distance.

The interplay of frequency-based and segmental cues in monolingual speech segmentation

In Experiment 2, we examined whether segmental information drove monolinguals' segmentation of new input, as found with bilinguals in Experiment 1. We presented two groups of Spanish 
monolinguals with the Spanish AL used in Experiment 1, and the German AL used by de la CruzPavía et al. (2015). Participants parsed the Spanish AL into a frequent-initial word order, but segmented the German AL into the opposite frequent-final parse. Spanish monolinguals hence showed similar segmentation preferences to their bilingual counterparts. Both ALs contained the same frequency-based information, which signaled a frequent-initial parse to these native speakers of a VO, functor-initial language. In the Spanish AL, frequency-based and segmental information provided convergent cues towards a frequent-initial segmentation. The frequent-final segmentation obtained in the group exposed to the German AL evidences that Spanish monolinguals relied on segmental cues to parse this AL. Further, these unfamiliar segmental cues overrode the conflicting but familiar frequency-based information present in the AL. Within the range of available phrase segmental cues, adult listeners - monolingual and bilingual — appear to weigh segmental cues more heavily than statistical cues.

This hierarchical arrangement of available phrase segmentation cues tallies with the results obtained in de la Cruz-Pavía et al. (2019), in which English monolinguals rely on unfamiliar prosodic cues to segment a structurally identical AL, disregarding familiar frequency-based cues. Taken together, these results suggest a hierarchy of segmentation cues in which segmental cues and prosodic cues are favored, while statistical cues are relegated to a secondary role. The relative position of segmental and prosodic cues is not known at present, and will be the object of future studies.

It is yet to be determined what specific segmental information biased Spanish monolinguals - as well as Basque-Spanish bilinguals in de la Cruz Pavía et al. (2015) — towards segmenting the German AL into the unfamiliar frequent-final order. Here, we propose that a specific type of subphonetic information might provide a plausible explanation for this bias, namely differences in the realization of voiceless stops in German and Spanish. The period between the 
explosion of the stop and the beginning of the vocal-fold vibration in the following vowel is known as the Voice Onset Time (VOT). VOT is negative when vibration of the vocal cords precedes the release of the plosive, and positive when this vibration follows the release of the plosive (Gussenhoven \& Jacobs, 1998). In Spanish, vibration precedes release in voiced stops, resulting in negative VOT values, while near simultaneous release of the plosive and vibration is found in voiceless stops, resulting in small, positive VOT values (average of $/ \mathrm{b}, \mathrm{d}, \mathrm{g} /:-69 \mathrm{~ms}, / \mathrm{p}, \mathrm{t}, \mathrm{k} /: 14$ ms, Castañeda, 1986³). In German, both voiced and voiceless stops have positive VOT values, and these can be up to three times longer in voiceless stops (average of $/ \mathrm{b}, \mathrm{d}, \mathrm{g} / \mathrm{:} 16 \mathrm{~ms}$, and $/ \mathrm{p}, \mathrm{t}$, kl: $51 \mathrm{~ms}$, Braunschweiler, 1997). We measured the VOT of the voiceless stops in Experiment 2's German and Spanish ALs (i.e., (/p, t, k/), and confirmed that values of voiceless stops were much greater in German (mean: $33 \mathrm{~ms}$ ) than Spanish (mean: $12 \mathrm{~ms}$ ).

Adult listeners discriminate very small differences in VOT values, even when the resulting contrast is not phonemic in their native language (Pisoni, Aslin, Percy, \& Hennessy, 1982). We hypothesize that the German AL's voiceless stops sound extremely hyperarticulated to the Spanish monolinguals, as if pronounced in emphatic speech. This hyperarticulation may have induced participants to perceive a phonological boundary in the signal. Segmental strengthening occurring at left edge of prosodic constituents is an acoustic cue to prosodic boundaries, especially at higher levels of the prosodic structure (Fougeron \& Keating, 1997; Cho \& Keating, 2001). Importantly, longer VOTs are often reported as strengthening cues (Jun, 1993; Cho \& Keating, 2001). Since only infrequent syllables in German AL contained voiceless stops, we speculate that Spanish listeners might have segmented the ambiguous stream at the onset of the syllables containing

${ }^{3}$ Castañeda (1986) reports a mean VOT value for each of the six stops found in Spanish. Braunschweiler (1997) reports only two VOT values per type of stop in German: voiced vs. voiceless. One value refers to the release before long vowels, the other before short vowels. For comparability, we report here the mean VOT value of each type of stop - voiced vs. voiceless - averaging across contexts and stops that belong to the same type. 
these consonants, inducing the observed frequent-final segmentation bias. Future studies will put to test this hypothesis.

\section{Conclusions}

In two experiments with four groups of highly proficient Basque-Spanish bilinguals and two groups of Spanish monolinguals, we show that segmental cues are available to adult bi- and monolinguals in order to parse phrases from new input. Segmental information determines bilinguals' choice of a strategy when parsing new input, though their segmentation is additionally modulated by a pragmatic factor, namely the language of context. The context language effect found in L1Basque bilinguals does not extend to L1Spanish bilinguals, and the cause for this asymmetry is currently unknown. Finally, the results of the present research show that the available phrase segmentation cues are arranged hierarchically, and that adult monolinguals disregard statistical cues in favor of segmental information when the two are in conflict. Future studies will determine the segmental cues - phonemic and subphonemic - available to adult listeners in speech segmentation.

\section{Acknowledgements}

We wish to thank Adam Zawiszewski and Idoia Ros for their help running a subset of the participants. This work was supported by the Basque Government under Grants [BFI07.253, IT1169-19 and IT1396-19], the Spanish Ministry of Science and Innovation under Grants [FFI201564183-P and FFI2016-80021-P], the French Investissements d'Avenir - Labex EFL Program under Grant [ANR-10-LABX-0083], the Agence Nationale de la Recherche [SpeechCode-ANR-15CE37-0009-01], and the European Research Council under Consolidator Grant [773202 ERC2017-COG 'BabyRhythm']. 


\section{References}

Alternberg, E. P., \& Cairns, H. S. (1983). The effects of phonotactic constraints on lexical processing in bilingual and monolingual subjects. Journal of Verbal Learning and Verbal Behavior 22(2), 174-188. https://doi.org/10.1016/S0022-5371(83)90134-2

Braunschweiler, N. (1997). Integrated cues of voicing and vowel length in German: A production $\begin{array}{lllll}\text { study. } \quad \text { Language and } & \text { Speech, } 40 & \text { (4), }\end{array}$ https://doi.org/10.1177/002383099704000403

Castañeda, M. L. (1986). El V.O. T. de las oclusivas sordas y sonoras españolas [The V.O.T. of the Spanish voiced and voiceless stops]. Estudios de fonética experimental II, 91-108.

Cho, T., \& Keating, P. 2001. Articulatory strengthening at the onset of prosodic domains in Korean. Journal of Phonetics 28, 155-190. https://doi.org/10.1006/jpho.2001.0131

Christensen, R. H. B. (2019). ordinal - Regression Models for Ordinal Data. R package version 2019.4-25. http://www.cran.r-project.org/package=ordinal/.

Colomé, À. (2001). Lexical activation in bilinguals' speech production: Language-specific or language-independent? Journal of Memory and Language, 45(4), 721-736. https://doi.org/10.1006/jmla.2001.2793

Costa, A., Caramazza, A., \& Sebastián-Gallés, N. (2000). The cognate facilitation effect: implications for models of lexical access. Journal of Experimental Psychology: Learning, Memory, and Cognition, 26(5), 1283. https://doi.org/10.1037/0278-7393.26.5.1283

Cutler, A. (1993). Phonological cues to open-and closed-class words in the processing of spoken sentences. Journal of Psycholinguistic Research, 22(2), 109-131. https://doi.org/10.1007/BF01067827

de la Cruz-Pavía, I., Elordieta, G., Sebastián-Gallés, N., \& Laka, I. (2015). On the role of frequency-based cues in the segmentation strategies of adult OV-VO bilinguals. 
International Journal of Bilingual Education and Bilingualism, 18, 225-241. https://doi.org/10.1080/13670050.2014.904839

de la Cruz-Pavía, I., Werker, J. F., Vatikiotis-Bateson, E., \& Gervain, J. (2019). Finding Phrases:

The Interplay of Word Frequency, Phrasal Prosody and Co-speech Visual Information in Chunking Speech by Monolingual and Bilingual Adults. Language and Speech, https://doi.org/10.1177/0023830919842353

Díaz, B., Erdocia, K., de Menezes, R. F., Mueller, J. L., Sebastián-Gallés, N., \& Laka, I. (2016). Electrophysiological Correlates of Second-Language Syntactic Processes Are Related to Native and Second Language Distance Regardless of Age of Acquisition. Frontiers in Psychology, 7. https://doi.org/10.3389/fpsyg.2016.00133

Duñabeitia, J. A., Cholin, J., Corral, J., Perea, M., \& Carreiras, M. (2010). SYLLABARIUM: An online application for deriving complete statistics for Basque and Spanish orthographic syllables. Behavior Research Methods 42, 118-125. https://doi.org/10.3758/BRM.42.1.118

Dutoit, T. (1997). An introduction to text-to-speech synthesis, Dordrecht, Netherlands: Kluwer.

Erdocia, K., Zawiszewski, A., \& Laka, I. (2014). Word Order Processing in a Second Language: From VO to OV. Journal of Psycholinguistic Research, 43(6), 815-837. https://doi.org/10.1007/s10936-013-9280-4

Finn, A. S., \& Hudson Kam, C. L. (2008). The curse of knowledge: First language knowledge impairs adult learners' use of novel statistics for word segmentation. Cognition, 108, 477499. https://doi.org/10.1016/j.cognition.2008.04.002

Fougeron, C., \& Keating, P. 1997. Articulatory strengthening at edges of prosodic domains. Journal of the Acoustical Society of America 101, 3728-3740. https://doi.org/10.1121/1.418332 
Forster, K. I., \& Forster, J. C. (2003). DMDX: A Windows display program with millisecond accuracy. Behavior Research Methods, Instruments, \& Computers, 35(1), 116-124. https://doi.org/10.3758/BF03195503

Gervain, J., Nespor, M., Mazuka, R., Horie, R., \& Mehler, J. (2008). Bootstrapping word order in prelexical infants: A Japanese-Italian cross-linguistic study. Cognitive Psychology, 57(1), 56-74. https://doi.org/10.1016/j.cogpsych.2007.12.001

Gervain, J., Sebastián-Gálles, N., Díaz, B., Laka, I., Mazuka, R., Yamane, N., Nespor, M., \& Mehler, J. (2013). Word frequency cues word order in adults: Crosslinguistic evidence. Frontiers in Psychology, 4, 689. https://doi.org/10.3389/fpsyg.2013.00689

Green, D. W. (1998). Mental control of the bilingual lexico-semantic system. Bilingualism: Language and Cognition, 1(2), 67-81. http://doi.org/10.1017/S1366728998000133

Grosjean, F. (2001). The Bilingual's Language Modes. In J. L. Nicol (Ed.), Explaining linguistics. One mind, two languages: Bilingual language processing (pp. 1-22). Malden: Blackwell Publishing.

Gussenhoven, C, \& Jacobs, H. (1998). Understanding phonology. London, UK: Arnold.

Harzing, A.-W. (2006). Response Styles in Cross-national Survey Research: A 26-country Study. International Journal of Cross Cultural Management, 6(2), 243-266. http://dx.doi.org/10.1177/1470595806066332

Hualde, J. I. (2003). Segmental phonology. In J. I. Hualde \& J. Ortiz de Urbina (Eds.), A grammar of Basque (pp. 15-64). Berlin, Germany: Mouton de Gruyter.

Hualde, J. I. (2005). The sounds of Spanish. New York City: Cambridge University Press.

Jun, S-A. (1993). The phonetics and phonology of Korean prosody, Ph.D. dissertation, Ohio State University [Published in 1996 by New York: Garland]. 
Luk, G., \& Bialystok, E. (2013). Bilingualism Is Not a Categorical Variable: Interaction between Language Proficiency and Usage. Journal of Cognitive Psychology, 25, 605-621. https://doi.org/10.1080/20445911.2013.795574

Marian, V., Blumenfeld, H. K., \& Kaushanskaya, M. (2007). The Language Experience and Proficiency Questionnaire (LEAP-Q): assessing language profiles in bilinguals and multilinguals. Journal of Speech, Language and Hearing Research, 50(4), 940-67. https://doi.org/10.1044/1092-4388(2007/067)

Marino, C., Bernard, C., \& Gervain, J. (accepted). Word frequency is a cue to lexical category at 8 months. Current Biology.

Mattys, S. L., White, L., \& Melhorn, J. F. (2005). Integration of multiple speech segmentation cues: A hierarchical framework. Journal of Experimental Psychology: General, 134, 477-500. https://doi.org/10.1037/0096-3445.134.4.477

Mersad, K., \& Nazzi, T. (2011). Transitional probabilities and positional frequency phonotactics in a hierarchical model of speech segmentation. Memory and Cognition, 39, 1085-1093. https://doi.org/10.3758/s13421-011-0074-3

Nespor, M., Shukla, M., van de Vijver, R., Avesani, C., Schraudolf, H., \& Donati, C. (2008). Different phrasal prominence realization in VO and OV languages. Lingue e Linguaggio, 7(2), 1-28.

Paap, K. R., Myuz, H. A., Anders, R. T., Bockelman, M. F., Mikulinsky, R., \& Sawi, O. M. (2017). No compelling evidence for a bilingual advantage in switching or that frequent language switching reduces switch cost. Journal of Cognitive Psychology, 29(2), 89-112. http://doi.org/10.1080/20445911.2016.1248436

Pisoni, D. B., Aslin, R. N., Perey, A. J., \& Hennessy, B. L. (1982). Some effects of laboratory training on identification and discrimination of voicing contrasts in stop consonants. Journal 
of Experimental Psychology: Human Perception and Performance, 8(2), 297-314. http://dx.doi.org/10.1037/0096-1523.8.2.297

Poepsel, T. J., \& Weiss, D. J. (2016). The influence of bilingualism on statistical word learning. Cognition, 152, 9-19. https://doi.org/10.1016/j.cognition.2016.03.001

Prior, A., \& Gollan, T. H. (2011). Good language-switchers are good task-switchers: Evidence from Spanish-English and Mandarin-English bilinguals. Journal of the International Neuropsychological Society, 17(4), 682-691. https://doi.org/10.1017/S1355617711000580

R Core Team (2019). R: A language and environment for statistical computing. R Foundation for Statistical Computing, Vienna, Austria. https://www.R-project.org/

Selkirk, E. (1996). The prosodic structure of function words. In J. L. Morgan \& K. Demuth (Eds.), Signal to syntax: Bootstrapping from speech to syntax in early acquisition (pp. 187-213). Hillsdale, USA: Erlbaum.

Shi, R., Morgan, J. L., \& Allopenna, P. (1998). Phonological and acoustic bases for earliest grammatical category assignment: a cross-linguistic perspective. Journal of Child Language, 25(1), 169-201. https://doi.org/10.1017/S0305000997003395

Smith, R., \& Hawkins, S. (2000). Allophonic influences on word-spotting experiments. In A. Cutler, J. M. McQueen and R. Zondervan (Eds.), Proceedings from References Workshop on Spoken Word Access Processes (SWAP) (pp. 139-142). Nijmegen, The Netherlands: Max-Planck-Institute for Psycholinguistics.

Soto-Faraco, S., Navarra, J., Weikum, W. M., Vouloumanos, A., Sebastián-Gallés, N., \& Werker, J. F. (2007). Discriminating languages by speech-reading. Perception \& Psychophysics, 69(2), 218-231. https://doi.org/10.3758/BF03193744

Tsui, A. S.-M., Erickson, L. C., Thiessen, E. D., \& Fennel, C. T. (2017). Statistical learning from accented speech: A bilingual advantage. In M. LaMendola \& J. Scott (Eds.), Proceedings 
of the 41st annual Boston University Conference on Language Development (BUCLD) (pp. 679-690). Somerville, MA: Cascadilla Press.

Verreyt, N., Woumans, E. V. Y., Vandelanotte, D., Szmalec, A., \& Duyck, W. (2016). The influence of language-switching experience on the bilingual executive control advantage. Bilingualism: Language and Cognition, 19(1), 181-190. http://doi.org/10.1017/S1366728914000352

Wang, T., \& Saffran, J. R. (2014). Statistical learning of a tonal language: the influence of bilingualism and previous linguistic experience. Frontiers in Psychology, 5. https://doi.org/10.3389/fpsyg.2014.00953

Weber, A., \& Cutler A. (2006). First-language phonotactics in second-language listening. Journal of the Acoustical Society of America 119(1), 597. https://doi.org/10.1121/1.2141003

Weikum, W. M., Vouloumanos, A., Navarra, J., Soto-Faraco, S., Sebastián-Gallés, N., \& Werker, J. F. (2013). Age-related sensitive periods influence visual language discrimination in adults. Frontiers in Systems Neuroscience, 7, 86. https://doi.org/10.3389/fnsys.2013.00086

Yim, O., \& Bialystok, E. (2012). Degree of conversational code-switching enhances verbal task switching in Cantonese-English bilinguals. Bilingualism: Language and Cognition, 15(4), 873-883. http://doi.org/10.1017/S1366728912000478

Zawiszewski, A., Gutiérrez, E., Fernández, B., \& Laka, I. (2011). Language distance and nonnative syntactic processing: Evidence from event-related potentials. Bilingualism: Language and Cognition, 14(03), 400-411. https://doi.org/10.1017/S1366728910000350

\section{Appendix 1. Characteristics of the new AL's CV lexicon}

The new lexicon contained only $\mathrm{CV}$ syllables that legally occur both in word-initial and wordinternal positions in Spanish and Basque. As a result, we excluded vibrants from the inventory, as 
in Basque these phonemes are illegal in word-initial position, and are only found in some recent borrowings from Spanish (Hualde, 2003). Frequency of occurrence of these syllables at word-initial position is hence extremely low in Basque, as we confirmed using the online application SYLLABARIUM (Type frequencies: $r a=6 ; r e=2 ; r i=1 ; r o=5 ; r u=1$. Token frequencies: $r a=12$; $r e=1 ; r i=1 ; r o=5 ; r u=1$, Duñabeitia et al., 2010; http://www.bcbl.eu/syllabarium/index.php). Although all L1Basque participants were highly proficient speakers of Spanish, a number of studies report interference of $L 1$ phonotactic constraints in the processing of input in the $L 2$ (e.g. Alternberg \& Cairns, 1983; Weber \& Cutler, 2006). Basque listeners could hence have been biased towards interpreting syllables containing vibrants as word-internal, unlike Spanish listeners.

In addition, we took into consideration the allophonic distribution characteristic of Basque and Spanish, excluding voiced stops from the lexicon. Voiced stops have two allophonic realizations in Spanish and Basque: (a) as a stop after a pause and after a nasal (and after /// for the voiced stop /d/), and (b) as an approximant at other positions (Hualde, 2003, 2005). No MBROLA voice available in Spanish includes this distinction. Importantly, allophonic variation is one of the available cues that assist adults in word segmentation (Smith \& Hawkins, 2000).

\section{Appendix 2. Subject-by-subject count of frequent-final responses out of 36 trials}

\section{Subject-by-subject count of frequent-final responses out of 36 trials}

\section{Basque-Spanish context Basque bilinguals}

Twenty-seven participants, mean of frequent-final responses: $13.67 / 36,37.96 \%$.

Part.1: 28; Part.2: 13; Part.3: 17; Part.4: 17; Part.5: 14; Part.6: 6; Part.7: 7; Part.8: 15; Part.9: 7;

Part.10: 11; Part.11: 6; Part.12: 30; Part.13: 17; Part.14: 9; Part.15: 16; Part.16: 4; Part.17: 1;

Part.18: 11; Part.19: 19; Part.20: 15; Part.21: 17; Part.22: 18; Part.23: 7; Part.24: 14; Part.25: 18; Part.26: 18; Part.27: 14. 


\section{Basque-Spanish context Spanish bilinguals}

Twenty-three participants, mean of frequent-final responses: $10.87 / 36,30.19 \%$.

Part.1: 12; Part.2: 5; Part.3: 12; Part.4: 15; Part.5: 9; Part.6: 10; Part.7: 8; Part.8: 15; Part.9: 15;

Part.10: 2; Part.11: 14; Part.12: 11; Part.13: 11; Part.14: 13; Part.15: 13; Part.16: 18; Part.17: 11;

Part.18: 14; Part.19: 14; Part.20: 4; Part.21: 13; Part.22: 1; Part.23: 10.

\section{Spanish-Basque context Basque bilinguals}

Twenty-one participants, mean of frequent-final responses: $12.81 / 36,35.58 \%$

Part.1: 6; Part.2: 23; Part.3: 7; Part.4: 8; Part.5: 16; Part.6: 11; Part.7: 12; Part.8: 24; Part.9: 11;

Part.10: 12; Part.11: 8; Part.12: 5; Part.13: 18; Part.14: 11; Part.15: 8; Part.16: 4; Part.17: 25;

Part.18: 9; Part.19: 20; Part.20: 16; Part.21: 15

\section{Spanish-Basque context Spanish bilinguals}

Twenty-one participants, mean of frequent-final responses: 12.52/36, 34.79\%

Part.1: 9; Part.2: 10; Part.3: 21; Part.4: 11; Part.5: 1; Part.6: 5; Part.7: 9; Part.8: 16; Part.9: 11;

Part.10: 18; Part.11: 8; Part.12: 12; Part.13: 18; Part.14: 15; Part.15: 25; Part.16: 4; Part.17: 6;

Part.18: 17; Part.19: 11; Part.20: 21; Part.21: 15.

\section{Basque-Spanish context Basque bilinguals - control group}

Twelve participants, mean of frequent-final responses: $13.75 / 36,38,19 \%$

Part.1: 9; Part.2: 24; Part.3: 3; Part.4: 17; Part.5: 15; Part.6: 26; Part.7: 4; Part.8: 9; Part.9: 4;

Part.10: 27; Part.11: 22; Part. 12: 5. 


\section{Spanish monolinguals - German AL}

Twenty-nine participants, mean of frequent-final responses: 25.00/36, $69.44 \%$

Part.1: 23; Part.2: 17; Part.3: 29; Part.4: 23; Part.5: 28; Part.6: 25; Part.7: 25; Part.8: 28; Part.9: 23;

Part.10: 23; Part.11: 32; Part.12: 20; Part.13: 34; Part.14: 22; Part.15: 27; Part.16: 29; Part.17: 21;

Part.18: 26; Part.19: 21; Part.20: 24; Part.21: 29; Part.22: 27; Part.23: 17; Part.24: 26; Part.25: 32;

Part.26: 14; Part.27: 34; Part.28: 17; Part.29: 29.

\section{Spanish monolinguals - Spanish/Basque AL}

Twenty-seven participants, mean of frequent-final responses: $14.70 / 36,40.84 \%$

Part.1: 9; Part.2: 15; Part.3: 28; Part.4: 21; Part.5: 11; Part.6: 17; Part.7: 15; Part.8: 11; Part.9: 14;

Part.10: 17; Part.11: 17; Part.12: 13; Part.13: 14; Part.14: 13; Part.15: 8; Part.16: 4; Part.17: 20;

Part.18: 28; Part.19: 12; Part.20: 18; Part.21: 7; Part.22: 8; Part.23: 14; Part.24: 22; Part.25: 13;

Part.26: 21; Part.27: 7. 
Figure 1: Basic structure of the ambiguous artificial language: the lexical categories and tokens of the language (upper panel), the two possible word orders of the ambiguous stream (middle panel), and three examples of the 36 test pairs (lower panel).

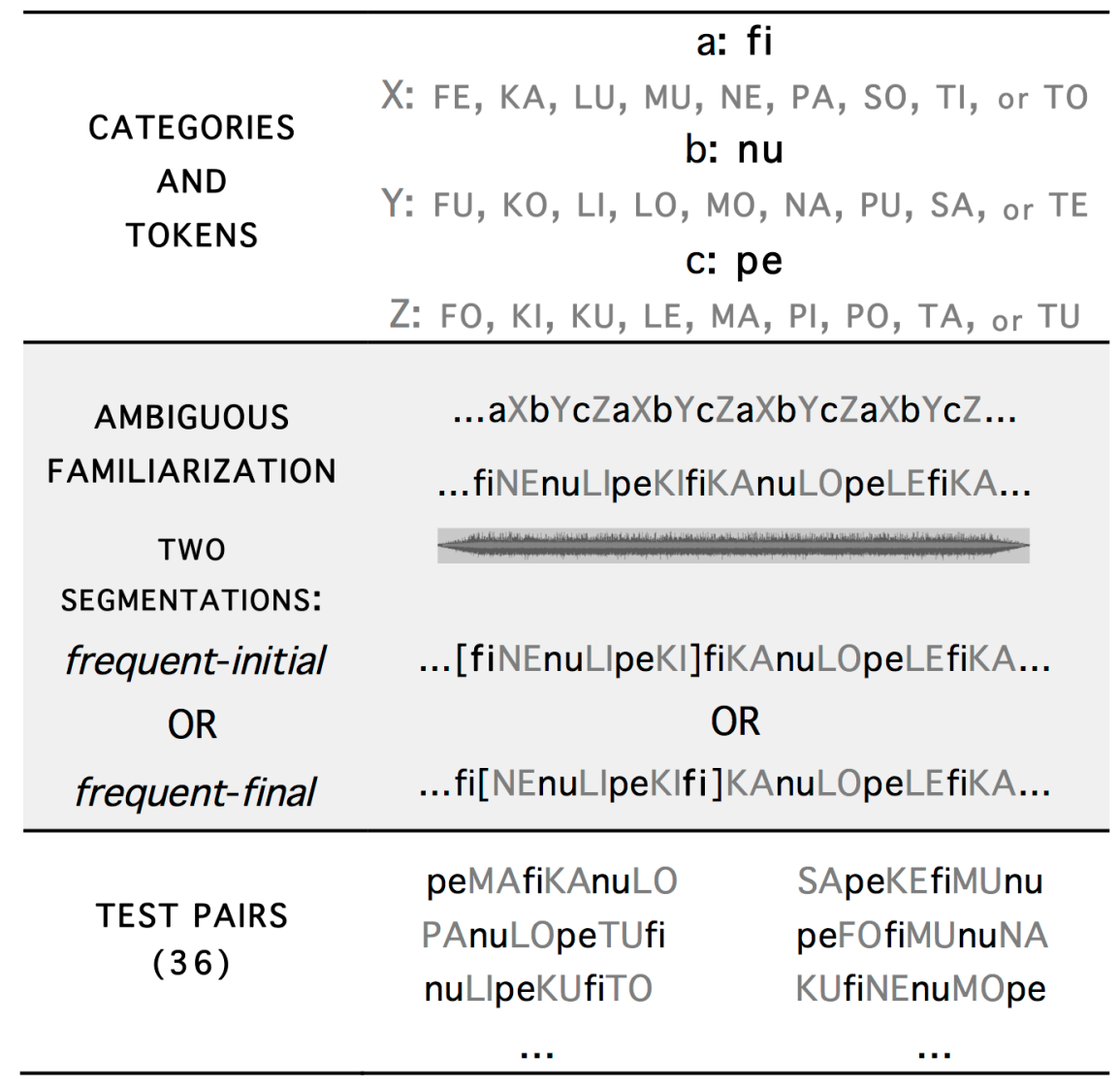


Table 1: Mean number of frequent-final responses out of the 36 test trials, standard error (SE), and 95\% confidence intervals (Cl) obtained in: the four groups of Basque-Spanish bilinguals tested in de la Cruz-Pavía et al. (2015) (marked by an + preceding the values), the four groups of BasqueSpanish bilinguals tested in Experiment 1, the control group of L1Basque bilinguals, and the two groups of Spanish monolinguals tested in Experiment 2.

\begin{tabular}{lll}
\hline \multicolumn{2}{c}{ NUMBER OF FREQUENT-FINAL RESPONSES OUT OF 36 TRIALS } \\
\hline & German Artificial Language & Spanish Artificial Language \\
\hline Bilinguals & & \\
L1Basque-L2Spanish - context Basque & $+28.10, \pm 1.42 \mathrm{SE}$ & $13.67, \pm 1.28 \mathrm{SE}$ \\
& $25.13-31.07$ & $11.03-16.30$ \\
L1Basque-L2Spanish - context Spanish & $+24.18, \pm 1.26 \mathrm{SE}$ & $10.87, \pm 0.91 \mathrm{SE}$ \\
& $21.60-26.75$ & $8.98-12.76$ \\
L1Spanish-L2Basque - context Basque & $+26.38, \pm 1.03 \mathrm{SE}$ & $12.81, \pm 1.38 \mathrm{SE}$ \\
& $24.28-28.48$ & $9.94-15.68$ \\
L1Spanish-L2Basque - context Spanish & $+26.81, \pm 1.27 \mathrm{SE}$ & $12.52, \pm 1.35 \mathrm{SE}$ \\
& $24.21-29.40$ & $9.70-15.34$ \\
Control & $\mathrm{n} / \mathrm{a}$ & $13.75, \pm 2.67 \mathrm{SE}$ \\
L1Basque-L2Spanish - context Basque & $7.88-19.62$ \\
Spanish Monolinguals & $25.00, \pm 0.96 \mathrm{SE}$ & $14.70, \pm 1.16 \mathrm{SE}$ \\
& $23.03-26.97$ & $12.32-17.08$ \\
\hline
\end{tabular}

+from de la Cruz-Pavía et al. (2015) 
Figure 2: Word order preferences of the four groups of Basque-Spanish bilinguals tested in Experiment 1, and the control group of L1Basque bilinguals. The bar graphs with standard error (top) depict the number and distribution of frequent-final responses out of the 36 test trials in the control group exposed to the 17-minute-long artificial language (leftmost bar), and the four groups exposed to the 9-minute-long artificial language (remaining bars). A mean below 18 (chance) indicates a frequent-initial segmentation preference, whereas a mean above 18 indicates a frequent-final segmentation preference. The boxplots (bottom) depict the distribution of participants' responses.

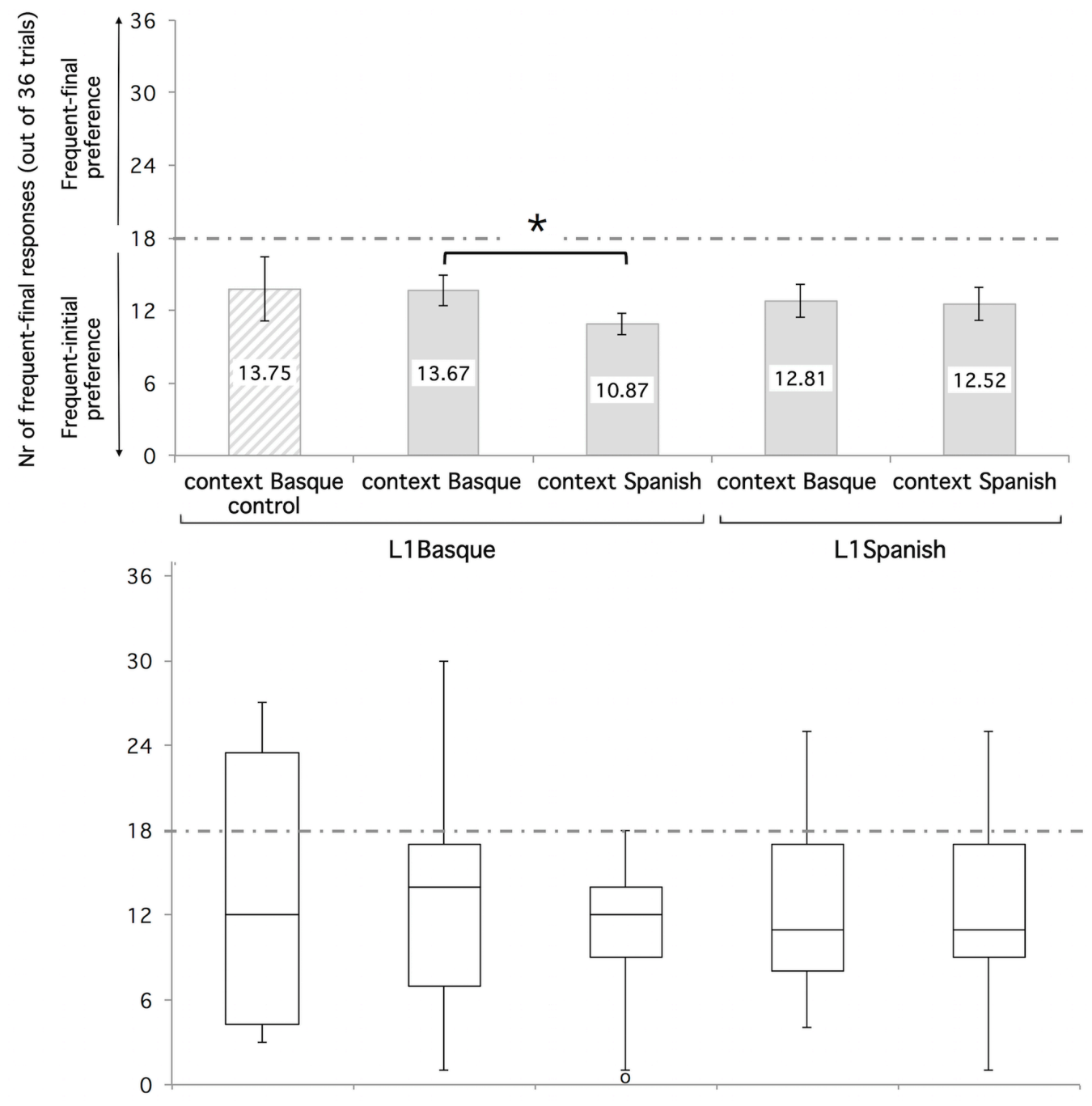


Table 2: Self-reported ratings of proficiency and language use of the four groups of bilinguals examined. Ratings were obtained from the language background questionnaire filled by Experiment 1's Basque-Spanish bilinguals. The table depicts mean proficiency (left) in the bilinguals' L2 - i.e., Basque for the L1Spanish bilinguals, and Spanish for the L1Basque bilinguals — and mean frequency of use of their two languages (right) in three points of development (i.e., infancy, adolescence and adulthood), followed by their standard deviation.

\begin{tabular}{|c|c|c|c|c|c|}
\hline \multicolumn{6}{|c|}{ LINGUISTIC BACKGROUND OF THE BILINGUAL PARTICIPANTS } \\
\hline & \multicolumn{2}{|c|}{$\begin{array}{c}\text { Proficiency in the L2 } \\
\text { [8-point Likert scale] }^{\star}\end{array}$} & \multicolumn{3}{|c|}{$\begin{array}{l}\text { Language use in situations other than at } \\
\text { school/university/work and home } \\
{[7 \text {-point Likert scale }]^{\star *}}\end{array}$} \\
\hline L1Basque context Basque & $\begin{array}{c}\text { Speak } \\
6.41,1.22\end{array}$ & $\begin{array}{l}\text { Understand } \\
7.26,0.59\end{array}$ & $\begin{array}{c}\text { Infancy } \\
1.74,0.98\end{array}$ & $\begin{array}{c}\text { Adolescence } \\
2.11,1.12\end{array}$ & $\begin{array}{l}\text { Adulthood } \\
2.48,1.28\end{array}$ \\
\hline L1Basque context Spanish+ & $6.27,0.94$ & $7.09,0.75$ & $2.04,0.82$ & $2.35,0.93$ & $2.52,0.67$ \\
\hline L1Spanish context Basque & $6.71,0.78$ & $7.33,0.66$ & $4.81,1.57$ & $4.76,1.34$ & $4.76,1.09$ \\
\hline L1Spanish context Spanish & $6.81,0.75$ & $7.71,0.46$ & $5.62,1.07$ & $5.62,1.02$ & $5.14,1.20$ \\
\hline
\end{tabular}


Figure 3: Basic structure of the ambiguous artificial language in de la Cruz-Pavía et al. (2015): the lexical categories and tokens of the languages (upper panel), the two possible word orders of the ambiguous stream (middle panel), and three examples of the 36 test pairs (lower panel).

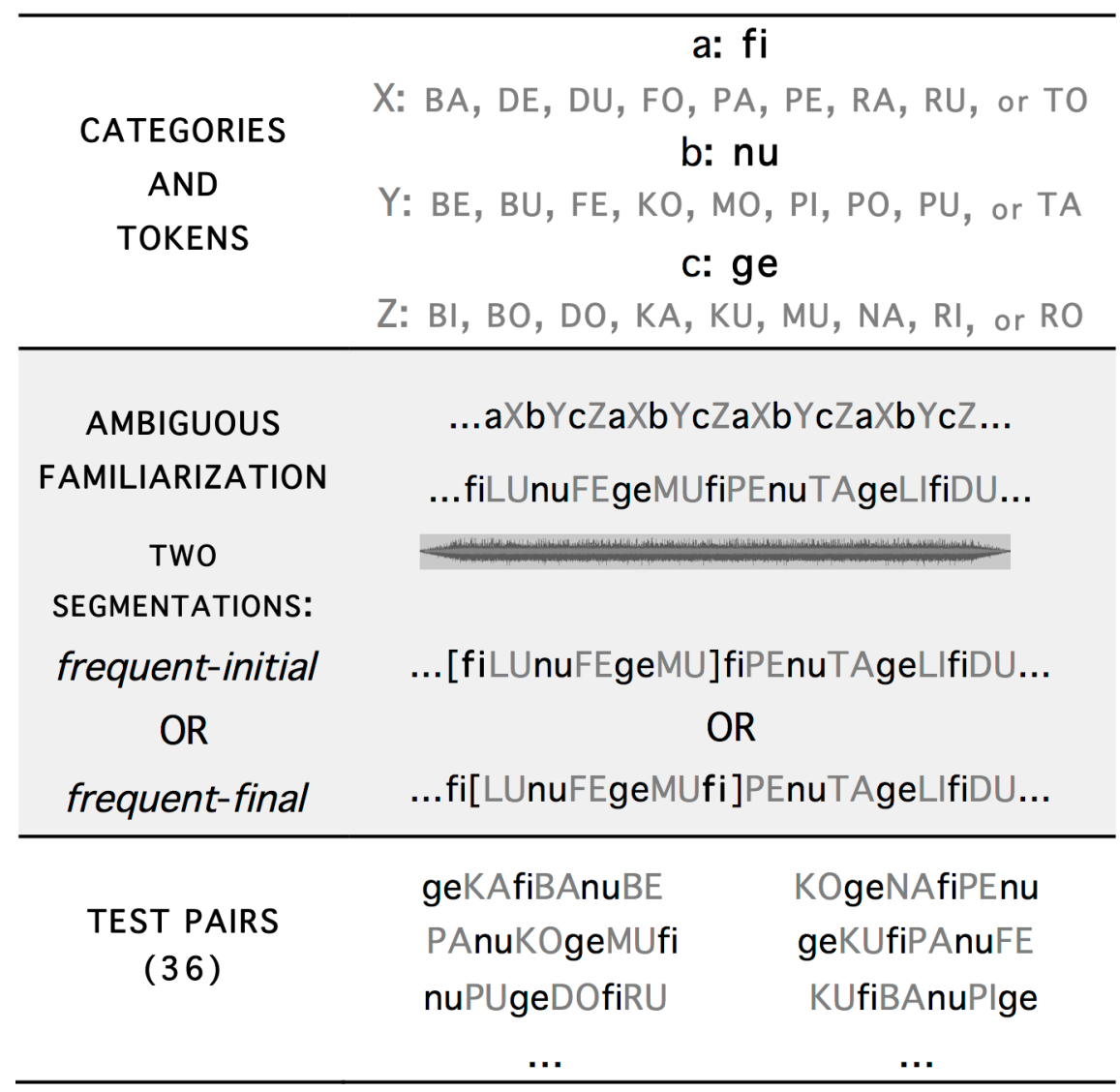


Figure 4: Word order preferences of the two groups of Spanish monolinguals. The bar graphs with standard error (top) and the boxplots (bottom) depict the mean number and distribution of frequentfinal responses out of the 36 test trials, in the groups exposed to the German (left) and the Spanish (right) artificial languages. A mean below 18 (chance) indicates a frequent-initial segmentation preference, whereas a mean above 18 indicates a frequent-final segmentation preference.
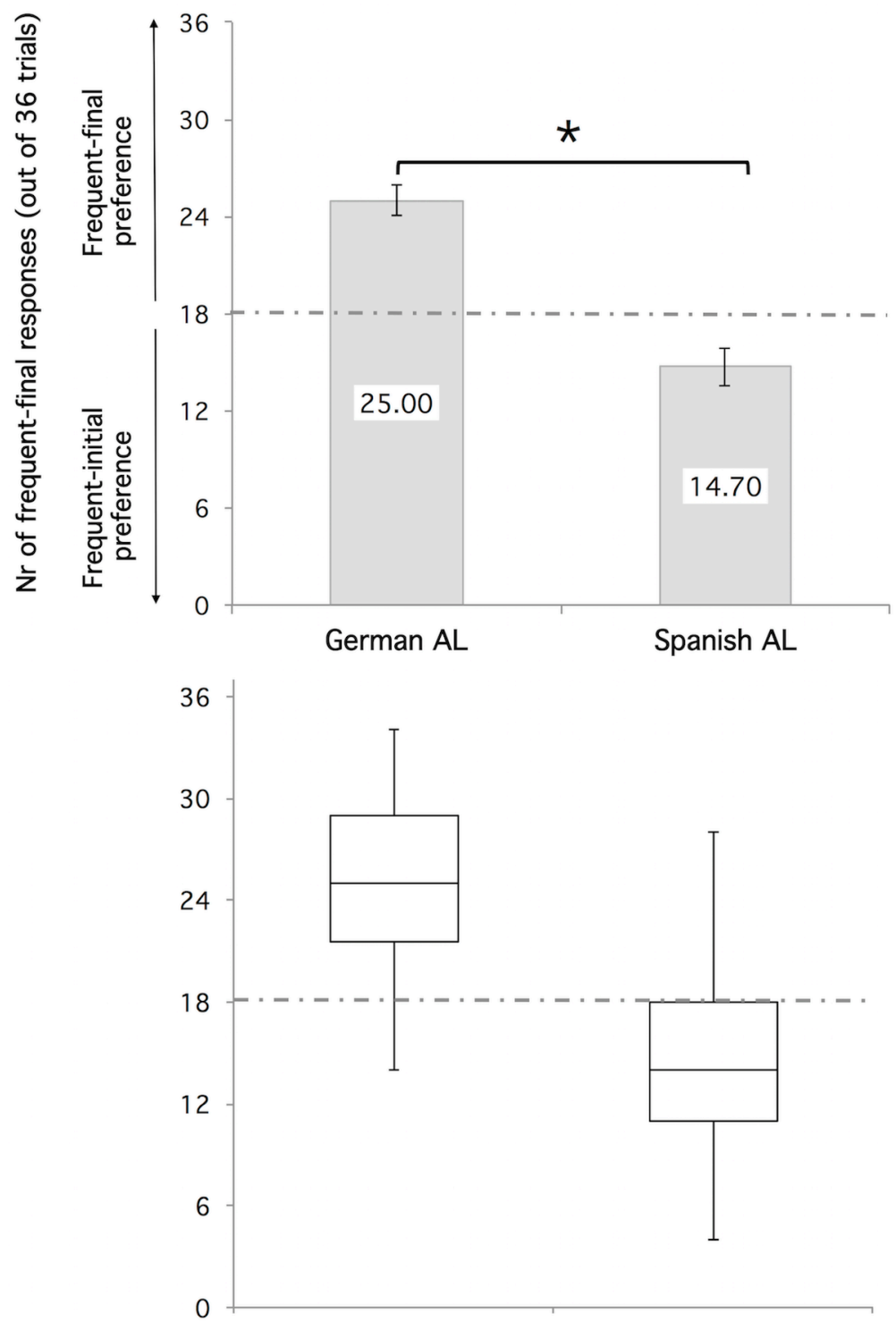